\title{
Recurrent and superior laryngeal nerve injury in thyroid surgery: literature review
}

\author{
Abdullatif Mahyoub ${ }^{1 *}$, Alaa A. Aljohani ${ }^{2}$, Abdullah J. Althobaiti ${ }^{3}$, Sami S. Alharbi ${ }^{4}$, \\ Abdulaziz A. Alahmary ${ }^{5}$, Raeed S. Algarni ${ }^{6}$, Sultanah K. Alamoudi ${ }^{7}$, Munirah S. Alshahrani ${ }^{8}$, \\ Turki M. Alkhaldi', Abdulhakim H. Alqubaishi ${ }^{6}$, Kaled A. Marzogi ${ }^{10}$
}

\author{
${ }^{1}$ Department of General Surgery, East Jeddah Hospital, Jeddah, Saudi Arabia \\ ${ }^{2}$ General Practitioner, King Faisal Hospital, Mecca, Saudi Arabia \\ ${ }^{3}$ College of Medicine, Taif University, Taif, Saudi Arabia \\ ${ }^{4}$ General Practitioner, King Abdulaziz Specialist Hospital, Taif, Saudi Arabia \\ ${ }^{5}$ General Practitioner, King Abdulaziz Medical City, National Guard Health Affairs, Riyadh, Saudi Arabia \\ ${ }^{6}$ College of Medicine, Imam Muhammad Ibn Saud Islamic University, Riyadh, Saudi Arabia \\ ${ }^{7}$ College of Medicine, Ibn Sina National College, Jeddah, Saudi Arabia \\ ${ }^{8}$ College of Medicine, King Khalid University, Abha, Saudi Arabia \\ ${ }^{9}$ Senior Registrar, Department of General Surgery, Prince Mohammed bin Abdulaziz Medical City, Aljouf, Saudi Arabia \\ ${ }^{10}$ College of Medicine, Umm Al-Qura University, Mecca, Saudi Arabia
}

Received: 23 December 2020

Revised: 27 December 2020

Accepted: 28 December 2020

\section{*Correspondence: \\ Dr. Abdullatif Mahyoub, \\ E-mail: amahyoub@moh.gov.sa}

Copyright: (c) the author(s), publisher and licensee Medip Academy. This is an open-access article distributed under the terms of the Creative Commons Attribution Non-Commercial License, which permits unrestricted non-commercial use, distribution, and reproduction in any medium, provided the original work is properly cited.

\begin{abstract}
Laryngeal nerve injury is considered one of the most common complications after thyroidectomy. It is associated with decreased quality of life because it will result in hoarseness of voice and aspiration. Identification of the risk factors and procedures to decrease the injury is crucial for handling laryngeal nerve injury. We searched the MEDLINE database using PubMed. Two independent reviewers reviewed the resulting papers and reviewed them based on our inclusion criteria. Based on the review results, the incidence of recurrent laryngeal nerve injury is higher than the external branch of the superior laryngeal nerve, but it is mainly due to under-reporting of the external branch of superior laryngeal nerve injury. Cancer surgery, surgeon experience, workload, re-operative procedures, and extent of surgery increased the incidence of the laryngeal nerve injury. Handling of these risk factors combined with visual dissection and inspection and/or intraoperative nerve monitoring decreased the incidence of the nerve injury. In conclusion, laryngeal nerve injury is a common post thyroidectomy complication. Anatomical dissection and visual inspection combined with intraoperative nerve monitoring is the most suitable option in high-risk thyroid surgeries.
\end{abstract}

Keywords: Recurrent laryngeal nerve, Thyroidectomy, Superior laryngeal nerve, Intraoperative nerve monitoring

\section{INTRODUCTION}

There is a world-wide increase in thyroid diseases in particular thyroid malignancy. In 2009, it was estimated that the incidence of thyroid cancer is 11.6 cases per 100000 in 2009 in the United States alone. Most of these cases are small papillary thyroid carcinoma. ${ }^{1,2}$ Thyroidectomy is the most successful and commonest procedure for various thyroid diseases and malignancies. ${ }^{2}$ However, thyroidectomy is associated with many complications that are associated with high morbidity. ${ }^{3}$ One of these complications is laryngeal nerve injury. Two 
laryngeal nerves are usually injured due to thyroidectomy namely the recurrent laryngeal nerve and external branch of the superior laryngeal nerve. The recurrent laryngeal nerve injury is the most reported nerve injury after thyroidectomy; it is mainly due to easy assessment during and after the procedure. Meanwhile, for the external branch of the superior laryngeal nerve, it is under-reported with a confusing incidence with no clear causes. ${ }^{2-4}$

Recurrent laryngeal nerve (RLN) and superior laryngeal nerve are both originated from the vagus nerve. The superior laryngeal nerve is close to the superior thyroid gland while the recurrent laryngeal nerve is more into the inferior part of the gland and in some cases is near the lateral part of the gland.,

Damage to the recurrent laryngeal nerve will result in posterior cricoarytenoid muscle affection that causes symptoms ranging from hoarseness to stridor and acute airway obstruction in bilateral damage. Permanent postoperative RLN injury occurs in approximately 0.3 to $3 \%$ of cases while transient injury can result in 3 to $8 \%$ of cases. $^{7}$

For the external branch of the superior laryngeal nerve injury, it innervates the cricothyroid muscle which is important for vocal cord tension which is responsible for the production of high pitch voice. ${ }^{8}$ Damage to the external branch of the superior laryngeal nerve will result in cricothyroid muscle impairment altering the voice and causes the inability to produce high-pitched sounds and in severe cases can cause microaspirations. ${ }^{6,8}$

Nowadays, there are modern techniques to decrease damage to laryngeal nerves. Visual inspection and dissection of nerves during procedures had decreased the incidence of damage in thyroidectomy patients. ${ }^{9,10}$ Moreover, intraoperative nerve monitoring combined with visual inspection and anatomical dissection had better results in high-risk patients. intraoperative nerve monitoring is a procedure where the assessment of the muscle innervated by the nerves is tested by electromyography. ${ }^{11}$ Other nerve monitoring techniques are used nowadays and are developed every day. However, visual inspection of the nerve is the gold standard for the diagnosis of the nerve injury. ${ }^{4}$

In this study, we aim to understand the incidence and risk factors for recurrent and superior laryngeal nerve injury in thyroidectomy and how it is affected after the introduction of different techniques.

\section{METHODS}

A literature search was performed in PubMed. We used the following search terms: ("laryngeal" OR "nerve injury") and ("thyroidectomy" or "thyroid cancer" or "thyroid surgery"). We included all studies that reported the epidemiology and risk factors for laryngeal nerve injuries in thyroidectomy including only superior and recurrent laryngeal nerve injury. No language or study design restrictions were included in the criteria.

\section{ANATOMICAL DISTRIBUTION AND PATTERNS OF RECURRENT AND SUPERIOR LARYNGEAL NERVE INJURY}

For recurrent laryngeal nerve, it also originates from the vagus nerve. However, it has different anatomy based on its laterality. The right recurrent laryngeal nerve passes around the subclavian artery and passes anterolateral to the tracheoesophageal groove. The left recurrent laryngeal nerve has a longer pathway passing around ligamentum arteriosum at the aortic arch ending in the tracheoesophageal groove. ${ }^{5}$

It is postulated that the left recurrent laryngeal nerve has more risk of injury during thyroidectomy compared to the right recurrent laryngeal nerve due to its longer course. However, another study reported that the right recurrent laryngeal nerve is injured more than the left recurrent laryngeal nerve. ${ }^{12}$ These claims are refuted by other studies that found no difference in the incidence of injury whether it is left or right. ${ }^{13,14}$ The most common site of injury was the final $2 \mathrm{~cm}$ of the extra laryngeal recurrent laryngeal nerve where it is covered by the tubercle of Zuckerkandl and fascial layers. ${ }^{15,16}$

For superior laryngeal nerve, it is originated from the vagus nerve and run for $10-20 \mathrm{~mm}$ before dividing it into external and internal branches. The external branch has a diameter of only $0.2 \mathrm{~mm}$ and after its pathway in the carotid sheath, it passes deep and posterior to the superior thyroid artery and in some cases, it passes lateral to it. For the internal branch, it did not have a significant role during thyroidectomy. ${ }^{6}$

Unlike the recurrent laryngeal nerve, it is hard to diagnose and estimate the incidence of superior laryngeal nerve injury. Several studies diagnosed the injury based on the clinical presentation of hoarseness of voice or laryngoscopy or both. ${ }^{6,17}$ However, these criteria for the diagnosis of the external branch of superior laryngeal nerve injury are not accurate and it was recommended to use electromyography, electroglottography, and fiberoptic stroboscopic laryngoscopy to diagnose the injury. Notwithstanding, these techniques are rarely used in clinical practice indicating underreporting of the external branch of superior laryngeal nerve injury. ${ }^{6,17}$

The incidence of the external branch of superior laryngeal nerve injury ranged from $0.9 \%$ up to $58 \%$ post thyroidectomy depending on the diagnostic procedures. Studies used electromyography had a high incidence of the external branch of superior laryngeal nerve injury compared to studies that depended on the clinical presentation and laryngoscope. ${ }^{8,18}$ After thyroidectomy, El-Guindy et al reported a rate of $2.4 \%$ after patients reported voice changes. ${ }^{17}$ Another study used the same 
technique and the external branch of the superior laryngeal nerve was diagnosed in $14 \%{ }^{8}$

Injury to recurrent laryngeal nerve results in the hoarseness of voice (of variable degrees), coughing, and microaspiration. ${ }^{5,19}$ Meanwhile, injury to the external branch of the superior laryngeal nerve increases the risk of aspiration and affects altering voice quality due to the inability to produce high-pitched sounds. ${ }^{6,8}$

Identification of risk factors for nerve injury and intraoperative procedures is crucial to decrease the laryngeal nerve injuries (Figure 1).

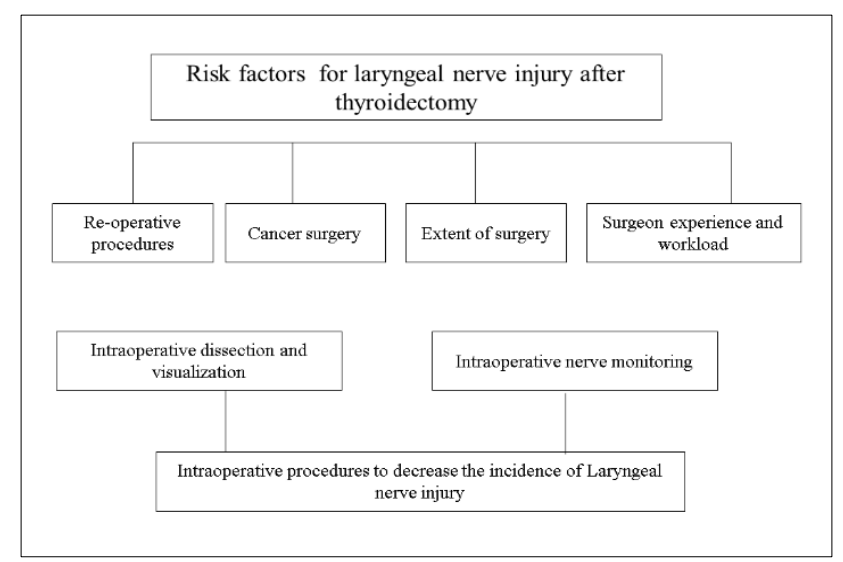

Figure 1: Figure summarizing the risk factors of laryngeal nerves and procedures to decrease it.

\section{RISK FACTORS FOR RECURRENT AND SUPERIOR LARYNGEAL NERVE INJURY DURING DISSECTION}

\section{Re-operative procedures}

There was an increased risk of recurrent laryngeal nerve injury in the case of repeated thyroid surgery. Current studies revealed an increased risk from 2 to $30 \%$ in any reoperative procedures. ${ }^{20}$ It is mainly because anatomical landmarks become distorted due to the scars and fibrous tissue formation from the previous procedures in this region. Besides, it will make it difficult for surgeons to dissect the nerve causing high-risk nerve injury. ${ }^{19,20}$ Jatzko et al compared the risk between recurrent laryngeal nerve injuries in primary surgery versus re-operative surgery and found that primary surgery had $0 \%$ risk for recurrent laryngeal nerve injury. Meanwhile, the risk in re-operative procedures is $8 \%$. Furthermore, $30 \%$ of cases, surgeons could not identify the recurrent laryngeal nerve site. ${ }^{19} \mathrm{~A}$ clinical trial reported an increased risk three times in secondary compared to primary benign goiter surgery. ${ }^{21}$

\section{CAUSE OF THYROIDECTOMY AND EXTENT OF SURGERY}

The recurrent laryngeal nerve is invaded in $20 \%$ of cases making it the most invaded soft tissue surrounding the thyroid gland. A study compared the risk of recurrent laryngeal nerve injury in benign thyroid diseases versus malignant thyroid diseases and found that there was $1.5 \%$ permanent recurrent laryngeal nerve injury compared to $0.5 \%$ in benign diseases. Moreover, it increased in secondary malignancy surgery up to $6 \%$. Another prospective cohort study found that there was a statistically significant rate of recurrent laryngeal nerve injury in malignant diseases that was $5 \%$ more than the benign tumor of the thyroid. This generally can be explained by the radical removal of the thyroid gland in case of thyroid malignancy compared to the conservative approach in benign thyroid tumors. Retrosternal goiter was another lesion that was associated with recurrent laryngeal nerve inury. ${ }^{22}$ For other benign lesions, there was no increased risk of recurrent laryngeal nerve injury after thyroidectomy. ${ }^{23}$

Extended resection always has a higher risk for recurrent laryngeal nerve injury compared to conservative resection. ${ }^{24}$ Examples of extended resection are lobectomy or total thyroidectomy. A study conducted on 3250 patients found a 2.6 times greater risk of recurrent laryngeal nerve injury for extended resection compared to a conservative approach. $^{3}$ Furthermore, another study found that the recurrent laryngeal nerve was higher in lobectomy compared to subtotal resection. ${ }^{22}$ That is why subtotal thyroidectomy is far more recommended than other approaches because surgeons leave less than $1 \mathrm{gm}$ of thyroid tissue near the recurrent laryngeal nerve at the ligament of berry sparing recurrent laryngeal nerve. ${ }^{25}$

\section{Surgeon workload and experience}

Few studies discussed the influence of surgeon experience and daily workload on the surgical outcome during thyroidectomy surgery. ${ }^{26-29}$ It was found that a workload of 100 thyroid cases per year was associated with better outcomes. $^{26}$ Besides, patients with re-operative and malignant diseases had better outcomes when the surgery was conducted by an experienced physician. ${ }^{29}$ However, other studies found that laryngeal nerve injury incidence was the same in both new and experienced surgeons. In addition, it was found that surgeons trained specifically for thyroidectomy had less incidence of laryngeal nerve injury compared to general surgeons who received nonspecific training. ${ }^{26-28}$

\section{PROCEDURES TO DECREASE THE RISK OF RECURRENT AND SUPERIOR LARYNGEAL NERVE INJURY}

\section{Intraoperative dissection and visualization}

Intraoperative dissection and visualization are considered a gold standard for any thyroid procedure. It helps preserve the nerve and protect it against any injury. For recurrent laryngeal nerve, a study found that the recurrent laryngeal nerve injury decreased more than half in cases where the nerve identified compared to it was not successfully 
identified. ${ }^{19}$ Another study found that visual identification had better outcomes compared to no visual inspection during thyroidectomy. ${ }^{30}$

The recurrent laryngeal nerve is usually dissected at the final $2 \mathrm{~cm}$ as it is the most common site of injury. Dissection of the recurrent laryngeal nerve from fascial layers containing branches of inferior thyroid artery. ${ }^{15}$ The correct dissection and visualization of the nerve during clamping of inferior thyroid vessels will protect the nerve. It was found that in the case of other anatomical courses of the nerve, there was an increased risk of recurrent laryngeal nerve injury specifically in cases of extra-laryngeal branched nerves. ${ }^{15,31}$

For the external branch of the superior laryngeal nerve, it is usually present in the superior thyroid pole, so it must be carefully dissected before ligation of superior thyroid arteries. It is usually dissected at the cricothyroid space. However other studies reported that the nerve is buried deep and it is unlikely to be injured. ${ }^{17,18}$ These studies found that there was no benefit for dissection compared to the recurrent laryngeal nerve. It was also found that unexpectedly, there was a less operative time in the case of the external branch of superior laryngeal nerve dissection and visualization compared to blindly perform the procedures. $^{6}$

\section{Intraoperative nerve monitoring}

A study found that visual identification combined with intraoperative nerve monitoring significantly decreased the risk of recurrent laryngeal nerve compared to visual inspection alone. ${ }^{32}$ It is reported a non-significant decrease of the nerve injury in case of the Intraoperative nerve monitoring compared to the significant efficacy of the anatomical dissection and nerve visualization during the thyroidectomy. ${ }^{19,33}$ However, it is recommended for the high-risk surgery to decrease the incidence of recurrent laryngeal nerve injury. ${ }^{10}$

In contrast to the recurrent laryngeal nerve, the external branch of the superior laryngeal nerve had benefitted from the introduction of intraoperative nerve monitoring. It had decreased the incidence of the external branch of superior laryngeal nerve injury. ${ }^{34}$ It is conducted through the electromyographic glottic response after the external branch of superior laryngeal nerve stimulation which helps the identification of $70-80 \%$ of patients with an external branch of superior laryngeal nerve stimulation. ${ }^{9,11,35}$ However, more improvement in the detection of the external branch of the superior laryngeal nerve is expected with the more development of newer electrode arrays and monitoring techniques.

\section{CONCLUSION}

In recent years, there is a decreased incidence of recurrent laryngeal nerve injury compared to the external branch of the superior laryngeal nerve after thyroidectomy operation.
Dealing with the risk factors associated with laryngeal nerve injury had significantly decreased the incidence of laryngeal nerve injury. More techniques are needed for more intraoperative visualization and testing of the laryngeal nerves.

Funding: No funding sources

Conflict of interest: None declared

Ethical approval: Not required

\section{REFERENCES}

1. Brito JP, Morris JC, Montori VM. Thyroid cancer: zealous imaging has increased detection and treatment of low risk tumours. BMJ. 2013;347:f4706.

2. Hu J, Zhao N, Kong R, Wang D, Sun B, Wu L. Total thyroidectomy as primary surgical management for thyroid disease: surgical therapy experience from 5559 thyroidectomies in a less-developed region. World J Surg Oncol. 2016;14(1):20.

3. Karamanakos SN, Markou KB, Panagopoulos K. Complications and risk factors related to the extent of surgery in thyroidectomy. Results from 2,043 procedures. Hormones (Athens, Greece). 2010;9(4):318-25

4. Horne SK, Gal TJ, Brennan JA. Prevalence and patterns of intraoperative nerve monitoring for thyroidectomy. Otolaryngol Head Neck Surg. 2007;136(6):952-6.

5. Myssiorek D. Recurrent laryngeal nerve paralysis: anatomy and etiology. Otolaryngol Clin North Am. 2004;37(1):25-44.

6. Morton RP, Whitfield P, Al-Ali S. Anatomical and surgical considerations of the external branch of the superior laryngeal nerve: a systematic review. Clin Otolaryngol.2006;31(5):368-74.

7. Zakaria HM, Al Awad NA, Al Kreedes AS. Recurrent laryngeal nerve injury in thyroid surgery. Oman Med J. 2011;26(1):34-8.

8. Aluffi P, Policarpo $\mathrm{M}$, Cherovac C, Olina M, Dosdegani R, Pia F. Post-thyroidectomy superior laryngeal nerve injury. Eur Arch Oto-rhino-Laryngol. 2001;258(9):451-4.

9. Uludag M, Aygun N, Kartal K. Contribution of intraoperative neural monitoring to preservation of the external branch of the superior laryngeal nerve: a randomized prospective clinical trial. Langenbeck's Arch Surg. 2017;402(6):965-76.

10. Yarbrough DE, Thompson GB, Kasperbauer JL, Harper CM, Grant CS. Intraoperative electromyographic monitoring of the recurrent laryngeal nerve in reoperative thyroid and parathyroid surgery. Surgery. 2004;136(6):1107-15.

11. Barczyński M, Konturek A, Stopa M, Honowska A, Nowak W. Randomized controlled trial of visualization versus neuromonitoring of the external branch of the superior laryngeal nerve during thyroidectomy. World J Surg. 2012;36(6):1340-7.

12. Lo CY, Kwok KF, Yuen PW. A prospective evaluation of recurrent laryngeal nerve paralysis 
during thyroidectomy. Arch Surg. 2000;135(2):2047.

13. Thomusch O, Sekulla C, Walls G, Machens A, Dralle $H$. Intraoperative neuromonitoring of surgery for benign goiter. Am J Surg. 2002;183(6):673-8.

14. Dionigi G, Boni L, Rovera F, Rausei S, Castelnuovo P, Dionigi R. Postoperative laryngoscopy in thyroid surgery: proper timing to detect recurrent laryngeal nerve injury. Langenbecks Arch Surg. 2010;395(4):327-31.

15. Serpell JW. New operative surgical concept of two fascial layers enveloping the recurrent laryngeal nerve. Ann Surg Oncol. 2010;17(6):1628-36.

16. Reeve T, Thompson NW. Complications of thyroid surgery: how to avoid them, how to manage them, and observations on their possible effect on the whole patient. World J Surg. 2000;24(8):971-5.

17. El-Guindy A, Abdel-Aziz M. Superior laryngeal nerve preservation in peri-apical surgery by mobilization of the viscerovertebral angle. J Laryngol Otol. 2000;114(4):268-73.

18. Hunt CJ. The superior and inferior laryngeal nerve as related to thvroid surgery. Am Surgeon. 1961;27:548-52.

19. Jatzko GR, Lisborg PH, Müller MG, Wette VM. Recurrent nerve palsy after thyroid operations-principal nerve identification and a literature review. Surgery. 1994;115(2):139-44.

20. Levin KE, Clark AH, Duh QY, Demeure M, Siperstein AE, Clark OH. Reoperative thyroid surgery. Surgery. 1992;111(6):604-9.

21. Thomusch O, Machens A, Sekulla C. Multivariate analysis of risk factors for postoperative complications in benign goiter surgery: prospective multicenter study in Germany. World $\mathbf{J}$ Surg. 2000;24(11):1335-41.

22. Dralle H, Sekulla C, Haerting J. Risk factors of paralysis and functional outcome after recurrent laryngeal nerve monitoring in thyroid surgery. Surgery. 2004;136(6):1310-22.

23. Godballe C, Madsen AR, Sørensen CH. Risk factors for recurrent nerve palsy after thyroid surgery: a national study of patients treated at Danish departments of ENT Head and Neck Surgery. Eur Arch Otorhinolaryngol. 2014;271(8):2267-76.

24. Bilimoria KY, Bentrem DJ, Ko CY. Extent of surgery affects survival for papillary thyroid cancer. Ann Surg. 2007;246(3):375-81.

25. Acun Z, Comert M, Cihan A, Ulukent SC, Ucan B, Cakmak GK. Near-total thyroidectomy could be the best treatment for thyroid disease in endemic regions. Arch Surg. 2004;139(4):444-7.

26. Bothra S, Sabaretnam M, Kannujia A. Patient, thyroid, and surgeon related factors that make thyroidectomy difficult-cohort study. Ann Med Surg. 2020;49:14-8

27. D'Orazi V, Sacconi A, Trombetta S. May predictors of difficulty in thyroid surgery increase the incidence of complications? Prospective study with the proposal of a preoperative score. BMC Surgery. 2019;18(1):1-8.

28. Godballe C, Madsen AR, Sørensen CH. Risk factors for recurrent nerve palsy after thyroid surgery: a national study of patients treated at Danish departments of ENT Head and Neck Surgery. Eur Arch Oto-rhino-laryngol.2014;271(8):2267-76.

29. Tsai SH, Chien SC, Nguyen PA. Incidences of hypothyroidism associated with surgical procedures for thyroid disorders: A nationwide population-based study. Front Pharmacol. 2019;10:1-8.

30. Hermann M, Alk G, Roka R, Glaser K, Freissmuth M. Laryngeal Recurrent Nerve Injury in Surgery for Benign Thyroid Diseases: Effect of Nerve Dissection and Impact of Individual Surgeon in More Than 27,000 Nerves at Risk. Ann Surg. 2002;235:261-8.

31. Beneragama T, Serpell JW. Extralaryngeal bifurcation of the recurrent laryngeal nerve: a common variation. ANZ J Surg. 2006;76(10):928-31.

32. Bai B, Chen W. Protective Effects of Intraoperative Nerve Monitoring (IONM) for Recurrent Laryngeal Nerve Injury in Thyroidectomy: Meta-analysis. Scientific Rep. 2018;8(1):1-11.

33. Chiang F-Y, Lee K-W, Chen H-C. Standardization of intraoperative neuromonitoring of recurrent laryngeal nerve in thyroid operation. World J Surg. 2010;34(2):223-9.

34. Hurtado-López L-M, Díaz-Hernández PI, BasurtoKuba E, Zaldívar-Ramírez FR, Pulido-Cejudo A. Efficacy of Intraoperative Neuro-Monitoring to Localize the External Branch of the Superior Laryngeal Nerve. Thyroid. 2016;26(1):174-8.

35. Masuoka H, Miyauchi A, Higashiyama $T$. Prospective randomized study on injury of the external branch of the superior laryngeal nerve during thyroidectomy comparing intraoperative nerve monitoring and a conventional technique. Head Neck. 2015;37(10):1456-60.

Cite this article as: Mahyoub A, Aljohani AA, Althobaiti AJ, Alharbi SS, Alahmary AA, Algarni $\mathrm{RS}$, et al. Recurrent and superior laryngeal nerve injury in thyroid surgery: literature review. Int J Community Med Public Health 2021;8:862-6. 